\title{
AVALIAÇÃO DA EFICIÊNCIA DE ÍNDICES DE PERIGO METEOROLÓGICO DE INCÊNDIOS FLORESTAIS PARA O ESTADO DE MINAS GERAIS
}

\author{
REIS, Thaynara Lopes dos - thaynaralopes29@gmail.com \\ Universidade Federal de Viçosa / UFV \\ TORRES, Fillipe Tamiozzo Pereira-tamiozzo@ufv.br \\ Universidade Federal de Viçosa / UFV
}

\begin{abstract}
RESUMO: : Este trabalho foi realizado com o objetivo de definir os índices de perigo de incêndio mais eficientes para as cidades do estado de Minas Gerais que possuem Estação Meteorológica Convencional. Foram utilizados dados do Instituto Nacional de Meteorologia e do Banco de Dados de Queimadas. No primeiro, coletou-se dados de precipitação, temperatura máxima, temperatura média, evaporação, umidade relativa média e velocidade do vento média para as cidades estudadas. No segundo, coletou-se os dias em que ocorreram incêndios florestais. De posse desses dados, calculou-se os seguintes índices: FWI, Telicyn, Nesterov, FMA, FMA+, P-EVAP e EVAP/P. Para comparar os índices e definir o mais eficiente, foi utilizado os métodos Skill Score e Distância de Mahalanobis. Os resultados do Skill Score mostraram que, para $77 \%$ das cidades estudadas, o índice mais eficiente é o FWI. Este mesmo índice também foi o mais eficiente em $81 \%$ das cidades estudadas quando usamos a Distância de Mahalanobis.
\end{abstract}

PALAVRAS-ChAVE: Fogo; Clima; Susceptibilidade; FWI.

EVALUATION OF THE EFFICIENCY OF METEOROLOGICAL DANGER INDICES OF FORESTRY FIRE FOR THE STATE OF MINAS GERAIS

\begin{abstract}
This work was carried out with the objective of defining the most efficient fire hazard indexes for the cities of the State of Minas Gerais that have a Conventional Meteorological Station. Data from the National Institute of Meteorology and the Queimadas Databank were used. In the first one, rainfall, maximum temperature, mean temperature, evaporation, mean relative humidity and mean wind speed data were collected for the cities studied. In the second, the days of forest fires were collected. With these data, the following indexes were calculated: FWI, Telicyn, Nesterov, FMA, FMA +, P-EVAP and EVAP / P. To compare the indexes and define the most efficient, the Skill Score and Mahalanobis Distance methods were used. The results of the Skill Score showed that, for $77 \%$ of the cities studied, the most efficient index is FWI. This same index was also the most efficient in $81 \%$ of the cities studied when using the Mahalanobis Distance.
\end{abstract}

KEYWORDS: Fire; Climate; Susceptibility; FWI.

EVALUACIÓN DE LA EFICIENCIA DE ÍNDICES DE PELIGRO METEOROLÓGICO DE INCENDIOS FORESTALES PARA EL ESTADO DE MINAS GENERALES

RESUMEN: Este trabajo fue realizado con el objetivo de definir los índices de peligro de incendio más eficientes para las ciudades del estado de Minas Gerais que poseen Estación Meteorológica Convencional. Se utilizaron datos del Instituto Nacional de Meteorología y del Banco de Datos de Quemaduras. En el primero, se recogieron datos de precipitación, temperatura máxima, temperatura media, evaporación, humedad relativa media y velocidad del viento media para las ciudades estudiadas. En el segundo, se recogieron los días en que ocurrieron incendios forestales. En el caso de los datos, se calcularon los siguientes índices: FWI, Telicyn, Nesterov, FMA, FMA +, P-EVAP y EVAP / P. Para comparar los índices y definir el más eficiente, se utilizaron los métodos Skill Score y Distancia de Mahalanobis. Los resultados del Skill Score mostraron que, para el $77 \%$ de las ciudades estudiadas, el índice más eficiente es el FWI. Este mismo índice también fue el más eficiente en el $81 \%$ de las ciudades estudiadas cuando usamos la Distancia de Mahalanobis.

PALABRAS CLAVE: Fuego; Clima; Susceptibilidad; FWI. 


\section{INTRODUÇÃO}

Os incêndios são um importante fator de perturbação que influenciam os ecossistemas florestais. Eles têm um forte impacto tanto sobre as condições bióticas como as abióticas (ADÁMEK et al., 2015). Eles são um componente essencial para o funcionamento de muitos ecossistemas. As últimas décadas, no entanto, trouxeram um aumento significativo nas ocorrências de incêndios em muitas áreas do mundo. Que resulta em um desequilíbrio entre os episódios de fogo e a recuperação do ecossistema, levando à fragmentação da paisagem e sua degradação. Além disso, os incêndios florestais são uma importante fonte de emissões de gases do efeito estufa (GEEs), sobretudo do dióxido de carbono, que ocasiona um efeito substancial sobre o estoque global (HUESCA et al., 2009).

Há três fatores principais que controlam o comportamento dos incêndios: o clima, os combustíveis e a topografia, sendo que o clima é considerado o mais dinâmico (JOLLY et al., 2015). Geralmente, as variáveis climáticas que interferem na ocorrência de incêndios são a precipitação, a umidade relativa do ar e a temperatura, que afeta a propagação do fogo indiretamente, através de sua influência sobre a umidade do combustível, e diretamente, através da direção do vento (ROTHERMEL, 1972; FINNEY, 2005). Com isso, a propagação do fogo é mais intensa quando o tempo está quente, seco e com vento (HOLSINGER et al., 2016).

Com uma quantidade limitada de equipamentos e recursos financeiros e humanos, os gestores florestais devem decidir a aplicação mais eficientes de subsídios em ações como a prevenção (ex. campanhas de educação ambiental), gestão de combustível (ex. queima prescrita e tratamentos mecânicos), présupressão (ex. planejamento e preparação, recrutamento e treinamento de bombeiros, manutenção de aceiros e pontos de água), supressão e medidas de restauração (MAVSAR et al., 2013).

Segundo Vijayakumar et al. (2015) o histórico de fogo tem um impacto significativo na forma como planos de manejo florestal e de conservação são dimensionados aumentando a sua eficiência.

É reconhecido que quanto mais se entender sobre o comportamento do fogo e sua resposta às condições ambientais, mais se poderá antecipar situações perigosas ou impactos de grande magnitude e mais acertadas serão as decisões sobre a gestão de combustíveis e dos incêndios (LINN et al., 2012).

Para se saber a probabilidade de ocorrência de um incêndio florestal, são utilizados índices de perigo de incêndio, tendo por base as condições atmosféricas diárias (SOARES, 1972a apud NUNES, 2005). Prever as condições de risco possibilita adotar medidas preventivas mais eficazes e econômicas (CHENEY, 1968 apud NUNES, 2005).

Portanto, o presente trabalho tem o objetivo de definir os índices de perigo de incêndio mais eficientes para as cidades do estado de Minas Gerais que possuem Estação Meteorológica Convencional.

\section{MATERIAL E MÉTODOS}

Para a realização deste trabalho, foi acessado o banco de dados do Instituto Nacional de Meteorologia (INMET) para coletar os dados diários no 
período de 01/01/2007 até 31/12/2016 de precipitação, temperatura máxima, temperatura média, evaporação, umidade relativa média e velocidade do vento média das cidades do estado de Minas Gerais que possuem Estação Meteorológica. Além disso, foi acessado o Banco de Dados de Queimadas (BDQueimadas) do Instituto Nacional de Pesquisas Espaciais (INPE) para coletar os dias que ocorreram incêndios e o número de focos em cada cidade estudada durante o período estudado.

Os índices de perigo de incêndio utilizados neste estudo foram: FWI (VAN WAGNER, 1987), Telicyn, Nesterov, FMA, FMA+, P-EVAP e EVAP/P (TORRES et al., 2017).

Os índices foram comparados com os métodos conhecidos como Skill Score e como Distância de Mahalanobis. O Skill Score se baseia em uma tabela de contingência (Tabela 1) que contém os valores observados e os valores previstos para um evento em uma população. O Skill Score é a razão da diferença entre os acertos na previsão e o número esperado de acertos e a diferença entre o número de dias observados e o número de dias com previsão de acertos (NUNES et al., 2010; TORRES et al., 2017). A Distância de Mahalanobis consiste em ordenar os valores diários dos índices de perigo em função do número de incêndios ocorridos. Esse método é calculado através da razão ao quadrado da diferença entre a média dos dias em que não houve a ocorrência de incêndios e a média dos dias em que houve a ocorrência de incêndios e o desvio padrão entre as duas médias (SAMPAIO, 1999).

Tabela 1 - Tabela de Contingência

\begin{tabular}{cccc}
\hline \multirow{2}{*}{ Evento } & \multicolumn{3}{c}{ Observado } \\
& & Incêndio & Não incêndio \\
\hline \multirow{2}{*}{ Previsto } & Incêndio & $\mathrm{a}$ & $\mathrm{b}$ \\
& Não incêndio & $\mathrm{c}$ & $\mathrm{d}$ \\
\hline
\end{tabular}

Em que, a e d são as previsões de sucesso; b e c são as previsões de insucesso do evento.

O número total de observações $(\mathrm{N})$ é determinado pela fórmula:

$$
\mathrm{N}=\mathrm{a}+\mathrm{b}+\mathrm{c}+\mathrm{d}
$$

O número de observações boas $(G)$ é determinado por:

$$
\mathrm{G}=\mathrm{a}+\mathrm{d}
$$

O número de previsões probabilísticas boas $(\mathrm{H})$ é representado por:

$$
H=N \cdot(1-p) \cdot(1-q)+N \cdot p \cdot q
$$

Em que, $\mathrm{p}=(\mathrm{a}+\mathrm{c}) / \mathrm{N}$ e $\mathrm{q}=(\mathrm{a}+\mathrm{b}) / \mathrm{N}$

\section{RESULTADOS E DISCUSSÃO}

\subsection{DESEMPENHO DOS ÍNDICES}

\section{SKILL SCORE}

Os resultados do Skill Score são apresentados na Tabela 2.

Tabela 2 - Skill Score para as cidades em estudo 


\begin{tabular}{|c|c|c|c|c|c|c|c|}
\hline Cidades & Telicyn & Nesterov & FMA & FWI & $\mathrm{FMA}+$ & P-EVAP & EVAP/P \\
\hline Aimorés & 0,015 & 0,013 & 0,018 & 0,061 & 0,025 & 0,033 & 0,022 \\
\hline Araçuai & 0,096 & 0,056 & 0,081 & 0,147 & 0,097 & 0,151 & 0,168 \\
\hline Araxá & 0,149 & 0,116 & 0,113 & 0,197 & 0,133 & 0,148 & 0,153 \\
\hline Arinos & 0,078 & 0,041 & 0,060 & 0,219 & 0,160 & 0,186 & 0,191 \\
\hline Bambui & 0,126 & 0,116 & 0,117 & 0,259 & 0,130 & 0,162 & 0,154 \\
\hline Barbacena & 0,052 & 0,042 & 0,053 & 0,175 & 0,060 & 0,091 & 0,065 \\
\hline Belo Horizonte & 0,045 & 0,031 & 0,037 & 0,058 & 0,052 & 0,051 & 0,054 \\
\hline Bom Despacho & 0,095 & 0,090 & 0,073 & 0,151 & 0,084 & 0,136 & 0,140 \\
\hline $\begin{array}{l}\text { Conceição do } \\
\text { Mato Dentro }\end{array}$ & 0,111 & 0,092 & 0,090 & 0,275 & 0,085 & 0,138 & 0,120 \\
\hline $\begin{array}{l}\text { Poços de } \\
\text { Caldas }\end{array}$ & 0,102 & 0,101 & 0,074 & 0,254 & 0,098 & 0,166 & 0,157 \\
\hline Caparaó & 0,017 & 0,017 & 0,013 & 0,073 & 0,017 & 0,027 & 0,028 \\
\hline Capinópolis & 0,021 & 0,020 & 0,019 & 0,187 & 0,154 & 0,156 & 0,155 \\
\hline Caratinga & 0,111 & 0,082 & 0,076 & 0,243 & 002 & 0,154 & 0,144 \\
\hline Carbonita & 0,056 & 0,039 & 0,045 & & & 0,053 & 0,065 \\
\hline $\begin{array}{l}\text { Coronel } \\
\text { Pacheco }\end{array}$ & 0,015 & 0,023 & 0,016 & 0,066 & 0,018 & 0,033 & 0,032 \\
\hline Curvelo & 0,048 & 0,011 & 0,123 & 0,160 & 0,143 & 0,164 & 0,174 \\
\hline Diamantina & 0,235 & 0,229 & 0,273 & 0,435 & 0,190 & 0,283 & 0,248 \\
\hline Divinópolis & 0,098 & 0,066 & 0,076 & 0,116 & 0,090 & 0,146 & 0,141 \\
\hline Espinosa & 0,010 & 0,005 & 0,005 & 0,047 & 0,038 & 0,047 & 0,053 \\
\hline Formoso & 0,129 & 0,108 & 0,128 & 0,195 & 0,134 & 0,150 & 0,148 \\
\hline Frutal & 0,249 & 0,213 & 0,261 & 0,329 & 0,273 & 0,283 & 0,301 \\
\hline Ibirité & 0,023 & 0,018 & 0,017 & 0,051 & 0,024 & 0,040 & 0,037 \\
\hline Itamarandiba & 0,124 & 0,099 & 0,094 & 0,239 & 0,098 & 0,138 & 0,135 \\
\hline Ituiutaba & 0,131 & 0,093 & 0,102 & 0,233 & 0,170 & 0,216 & 0,215 \\
\hline Janaúba & 0,127 & 0,093 & 0,109 & 0,131 & 0,111 & 0,146 & 0,155 \\
\hline Januária & 0,190 & 0,178 & 0,180 & 0,279 & 0,193 & 0,242 & 0,219 \\
\hline João Pinheiro & 0,185 & 0,130 & 0,229 & 0,328 & 0,289 & 0,269 & 0,279 \\
\hline Juiz de Fora & 0,151 & 0,126 & 0,114 & 0,292 & 0,116 & 0,210 & 0,227 \\
\hline Juramento & 0,019 & 0,018 & 0,013 & 0,021 & 0,012 & 0,019 & 0,021 \\
\hline Lavras & 0,065 & 0,048 & 0,053 & 0,112 & 0,062 & 0,125 & 0,100 \\
\hline Machado & 0,082 & 0,079 & 0,059 & 0,219 & 0,077 & 0,109 & 0,114 \\
\hline Monte Azul & 0,021 & 0,007 & 0,024 & 0,030 & 0,028 & 0,049 & 0,051 \\
\hline Montes Claros & 0,101 & 0,078 & 0,088 & 0,138 & 0,104 & 0,125 & 0,127 \\
\hline Paracatu & 0,348 & 0,329 & 0,346 & 0,424 & 0,350 & 0,370 & 0,370 \\
\hline Patos de Minas & 0,163 & 0,147 & 0,145 & 0,267 & 0,169 & 0,226 & 0,237 \\
\hline Pedra Azul & 0,073 & 0,088 & 0,050 & 0,194 & 0,085 & 0,175 & 0,194 \\
\hline Pirapora & 0,066 & 0,043 & 0,055 & 0,083 & 0,061 & 0,080 & 0,079 \\
\hline Pompeu & 0,192 & 0,134 & 0,146 & 0,222 & 0,168 & 0,206 & 0,221 \\
\hline Salinas & 0,117 & 0,078 & 0,087 & 0,169 & 0,096 & 0,148 & 0,150 \\
\hline Sete Lagoas & 0,087 & 0,067 & 0,069 & 0,120 & 0,082 & 0,135 & 0,127 \\
\hline Uberaba & 0,431 & 0,389 & 0,416 & 0,502 & 0,433 & 0,436 & 0,444 \\
\hline Unaí & 0,280 & 0,227 & 0,259 & 0,347 & 0,277 & 0,313 & 0,307 \\
\hline Viçosa & 0,038 & 0,027 & 0,023 & 0,101 & 0,028 & 0,054 & 0,056 \\
\hline
\end{tabular}

Ao observar a Tabela 2, percebe-se que: em 33 cidades o índice que melhor indicou os riscos de incêndios foi o FWI; em 7 cidades o melhor índice foi o EVAP/P; e em 3 cidades foi o P-EVAP. Vale ressaltar que na cidade Carbonita 
não foram calculados os índices FWI e FMA +, pois os dados de velocidade de vento não foram registrados para esta cidade.

Resultados distintos foram encontrados para outras regiões do Brasil como, por exemplo, Nunes et al. (2010) observou que o melhor índice para o Distrito Florestal de Monte Alegre foi o FMA+, onde foram obtidos os valores de 0,0517 e 0,1165 para FMA e FMA+, respectivamente. O mesmo índice foi o melhor avaliado por Borges et al. (2011) para o norte do estado do Espírito Santo, onde os valores variaram de 0,1626 a 0,2055 nas 5 estações meteorológicas utilizadas. Os primeiros autores avaliaram os índices FMA e FMA + e os segundos avaliaram o FMA, o FMA + e o Nesterov. Já Sampaio (1999), avaliando os índices Nesterov, Telicyn, FMA e FWI, encontrou que o melhor índice para a cidade de Agudos, localizada no estado de São Paulo, também foi o FWI, cujo valor foi de 0,1838.

Torres et al. (2017), avaliando o estado de São Paulo, destacou o FWI como o índice mais eficiente, seguido por Telicyn, Nesterov e FMA. Para o estado de Sergipe, White (2011) encontrou um valor de 0,36 para FMA+, um de 0,197 para o índice de Telicyn e um de 0,184 para FMA, sendo que foi definido o índice de Angstron $(0,582)$ como o mais eficiente para o estado. Na Bahia, White et al. (2015) definiram o índice de Telicyn como o mais eficiente, encontrando um valor de 0,1 , os valores de outros índices foram 0,08 para o índice de Angstron, de 0,05 para FMA+, 0,03 para Nesterov e 0,01 para FMA.

Em outros países como a Alemanha, o melhor índice também foi o FWI, sendo que foram avaliados o Nesterov e o FWI, entre outros (HOLSTEN et al. 2013). Já em Ticino, na Suíça, segundo Zumbrunnen et al. (2011), o Nesterov foi o mais eficiente.

\section{DISTÂNCIA DE MAHALANOBIS}

Os resultados da Distância de Mahalanobis são apresentados na Tabela 3.

Tabela 3 - Distância de Mahalanobis para as cidades em estudo

\begin{tabular}{cccccccc}
\hline Cidades & Telicyn & Nesterov & FMA & FMA & FWI & P-EVAP & EVAP/P \\
\hline Aimorés & 0,209 & 0,407 & 0,255 & 0,433 & 1,412 & 0,303 & 0,292 \\
Araçuai & 0,772 & 0,885 & 1,009 & 1,134 & 1,579 & 1,123 & 0,950 \\
Araxá & 1,152 & 1,448 & 1,363 & 1,492 & 2,442 & 1,474 & 1,422 \\
Arinos & 1,043 & 1,443 & 1,277 & 0,896 & 1,047 & 0,919 & 0,725 \\
Bambui & 0,446 & 0,674 & 0,578 & 0,729 & 1,107 & 0,666 & 0,572 \\
Barbacena & 0,871 & 0,696 & 0,369 & 0,414 & 2,010 & 0,476 & 0,393 \\
Belo Horizonte & 0,479 & 0,754 & 0,620 & 0,716 & 1,487 & 0,687 & 0,563 \\
Bom Despacho & 0,706 & 1,046 & 0,766 & 1,138 & 1,879 & 1,108 & 0,989 \\
Conceição do Mato & 0,959 & 1,450 & 0,854 & 0,977 & 2,523 & 1,230 & 0,975 \\
Dentro & 1,704 & 1,508 & 1,773 & 1,960 & 3,465 & 2,099 & 1,986 \\
Poços de Caldas & 2,584 & 3,019 & 2,736 & 3,063 & 4,412 & 3,363 & 3,258 \\
Caparaó & 0,594 & 0,732 & 0,677 & 0,568 & 0,978 & 0,627 & 0,696 \\
Capinópolis & & & & & &
\end{tabular}




\begin{tabular}{|c|c|c|c|c|c|c|c|}
\hline Caratinga & 0,670 & 1,548 & 0,745 & 1,010 & 3,016 & 1,434 & 1,103 \\
\hline Carbonita & 0,185 & 0,587 & 0,418 & & & 0,688 & 0,629 \\
\hline Coronel Pacheco & 0,251 & 6,007 & 0,564 & 2,432 & 5,985 & 5,695 & 0,963 \\
\hline Curvelo & 1,058 & 0,983 & 0,855 & 0,957 & 1,697 & 0,943 & 1,037 \\
\hline Diamantina & 0,780 & 1,119 & 0,866 & 1,222 & 1,779 & 1,182 & 1,063 \\
\hline Divinópolis & 1,965 & 2,843 & 2,581 & 2,826 & 2,664 & 3,008 & 2,789 \\
\hline Espinosa & 0,898 & 1,209 & 1,025 & 1,309 & 0,875 & 0,895 & 0,075 \\
\hline Formoso & 0,794 & 1,048 & 0,757 & 0,888 & 1,417 & 0,925 & 0,782 \\
\hline Frutal & 0,555 & 0,549 & 0,552 & 0,581 & 0,935 & 0,584 & 0,557 \\
\hline Ibirité & 5,950 & 6,135 & 5,678 & 5,468 & 4,767 & 6,582 & 5,211 \\
\hline Itamarandiba & 0,338 & 0,503 & 0,322 & 0,372 & 1,166 & 0,444 & 0,346 \\
\hline Ituiutaba & 0,642 & 0,758 & 0,755 & 0,786 & 1,101 & 0,645 & 0,609 \\
\hline Janaúba & 0,468 & 0,830 & 0,683 & 0,817 & 0,671 & 0,798 & 0,676 \\
\hline Januária & 0,002 & 0,002 & 0,000 & 1,020 & 1,182 & 0,962 & 0,848 \\
\hline João Pinheiro & 0,546 & 0,727 & 0,644 & 0,743 & 0,977 & 0,794 & 0,663 \\
\hline Juiz de Fora & 0,645 & 0,760 & 0,709 & 0,794 & 1,815 & 0,718 & 0,884 \\
\hline Juramento & 0,001 & 0,004 & 0,002 & 0,267 & 0,965 & 0,328 & 0,241 \\
\hline Lavras & 0,066 & 0,043 & 0,071 & 1,431 & 2,687 & 1,494 & 1,419 \\
\hline Machado & 0,610 & 0,883 & 0,764 & 0,870 & 2,214 & 0,821 & 0,765 \\
\hline Monte Azul & 1,932 & 1,678 & 2,398 & 2,294 & 1,338 & 2,089 & 1,847 \\
\hline Montes Claros & 0,002 & 0,007 & 0,002 & 0,875 & 0,910 & 0,859 & 0,768 \\
\hline Paracatu & 0,000 & 0,001 & 0,000 & 0,854 & 1,271 & 0,853 & 0,780 \\
\hline Patos de Minas & 0,009 & 0,001 & 0,006 & 1,086 & 1,792 & 1,101 & 0,935 \\
\hline Pedra Azul & 0,103 & 0,328 & 0,118 & 0,884 & 1,863 & 0,993 & 0,888 \\
\hline Pirapora & 1,087 & 1,257 & 1,126 & 1,208 & 1,622 & 1,231 & 0,896 \\
\hline Pompeu & 0,692 & 0,735 & 0,863 & 0,923 & 1,237 & 0,806 & 0,844 \\
\hline Salinas & 0,618 & 0,932 & 0,819 & 0,942 & 1,519 & 1,059 & 0,868 \\
\hline Sete Lagoas & 0,003 & 0,005 & 0,002 & 1,795 & 2,732 & 1,886 & 1,595 \\
\hline Uberaba & 0,019 & 0,018 & 0,024 & 0,911 & 1,496 & 0,863 & 0,932 \\
\hline Unaí & 0,831 & 0,971 & 0,909 & 0,947 & 1,336 & 0,934 & 0,868 \\
\hline Viçosa & 1,072 & 1,498 & 1,169 & 1,315 & 2,855 & 1,487 & 1,683 \\
\hline
\end{tabular}

Ao observar a Tabela 3, percebe-se que o índice que melhor indicou os riscos de incêndios em 35 cidades foi o FWI, em 3 cidades foi o Nesterov, em 1 foi o FMA, em 1 cidade foi o FMA + e em 3 cidades o melhor índice foi o P-EVAP. Como dito anteriormente, na cidade Carbonita não foram calculados os índices FWI e FMA +, pois os dados de velocidade de vento não foram registrados para esta cidade.

Um resultado similar foi encontrado por Sampaio (1999), avaliando os índices Nesterov, Telicyn, FMA e FWI para a cidade de Agudos, localizada no estado de São Paulo. Esse autor encontrou um valor médio da distância de 
Mahalanobis de 1,15 para o FWI; 0,97 para FMA e 0,92 para Nesterov e Telecyn.

Um outro trabalho desenvolvido por Viegas et al. (1994) teve como resultado uma distância de Mahalanobis variando entre 0,0 e 1,88 para o índice FWI. Esse trabalho foi desenvolvido para regiões da França, Itália e Portugal, sendo encontrado valores variando entre 0,0 e 1,61 para o índice francês; entre 0,04 e 1,43 para o índice italiano; e entre 0,23 e 1,42 para o índice português.

\subsection{AVALIAÇÃO DAS TÉCNICAS UTILIZADAS}

Ambos índices utilizam o número de dias de ocorrência e não ocorrência de incêndios como dados de entrada. Porém, segundo Schunk et al. (2017), técnicas como estas não podem ser universalmente aplicáveis, pois é possível que haja poucos incêndios registrados para suportar análises significativas.

No Brasil, a grande maioria dos incêndios é de origem antrópica (MÉLO et al. 2011), observando-se que, mesmo que o risco seja máximo, pode não ocorrer a presença de fogo, sendo o oposto também verdadeiro, em que pode ocorrer incêndio em dias classificados como nulo. Torres et al. (2014) afirma que não basta que os fatores diretamente associados à ocorrência dos incêndios florestais estejam favoráveis à ignição inicial e à propagação do fogo, também é necessária a chama inicial para dar início à reação de combustão, o que contribui para a afirmativa inicial. Porém, no Brasil, a única fonte de dados disponível é a por meio de satélites, não sendo possível o acréscimo da variável "ação antrópica" nos cálculos.

\section{CONCLUSÃO}

Com base nos resultados apresentados e discutidos, pode-se concluir que o melhor índice de perigo de incêndios florestais para as cidades de Minas Gerais foi o FWI, pois apresentou melhores resultados em aproximadamente $77 \%$ das cidades estudadas com base no Skill Score e, aproximadamente, $81 \%$ com base na Distância de Mahalanobis. Outros índices que apresentaram melhores valores foram o EVAP/P, O P-EVAP e o Nesterov, porém o número de cidades em que esses índices apresentaram melhores resultados não é significativo. Com isso, o índice de FWI pode ser aplicado para todo o estado de Minas Gerais.

\section{REFERÊNCIAS BIBLIOGRÁFICAS}

ADÁMEK M.; BOBEK P.; HADINCOVÁ; WILD J.; KOPECKÝ, M. Forest fires within a temperate landscape: a decadal and millennial perspective from a sandstone region in Central Europe. Forest Ecology and Management, v.336, p.81-90, 2015.

BORGES, T. S.; FIEDLER, N. C.; SANTOS, A. R.; LOUREIRO, E. B.; MAFIA, R. G. Desempenho de Alguns Índices de Risco de Incêndios em Plantios de Eucalipto no Norte do Espírito Santo. Floresta e Ambiente, 2011.

FINNEY, M. A. The challenge of quantitative risk analysis for wildland fire. Forest Ecology and Management, p. 211:97-108, 2005. 
HOLSINGER, L.; PARKS, S. A.; MILLER, C. Forest Ecology and Management Weather, fuels, and topography impede wildland fire spread in western US landscapes. Forest Ecology and Management, p. 380:59-69, 2016.

HOLSTEN, A; DOMINIC, A. R.; COSTA, L.; KROPP, J. P. Evaluation of the performance of meteorological forest fire indices for German federal states. Forest Ecology and Management, v.287, p. 123-131, 2013.

HUESCA, M.; LITAGO, J.; PALACIOS-ORUETA, A.; MONTES, F.; SEBASTIÁNLÓPEZ, A.; ESCRIBANO, P. Assessment of forest fire seasonality using MODIS fire potential: A time series approach. Agricultural and Forest Meteorology, v.149(11), 2009.

INMET - Instituto Nacional de Meteorologia. Banco de Dados Meteorológicos para Ensino e Pesquisa. Acesso em março/2017. Disponível em: http://www.inmet.gov.br/portal/estacoes Convencionais

INPE - Instituto Nacional de Pesquisas Espaciais. Banco de Dados de Queimadas - BDQueimadas. Acesso em maio/2017. Disponível em: https://prodwwwqueimadas.dgi.inpe.br/bdqueimadas/

JOLLY, W. M.; COCHRANE, M. A.; FREEBORN, P. H.; HOLDEN, Z. A.; BROWN, T. J.; WILLIAMSON, G. J.; et al. Climate-induced variations in global wildfire danger from 1979 to 2013. Nature Communications. 2015.

LINN, R. R.; CANFIELD, J. M.; CUNNINGHAM, P.; EDMINSTER, C.; DUPUY, J. L.; PIMONT, F. Using periodic line fires to gain a new perspective on multidimensional aspects of forward fire spread. Agricultural and Forest Meteorology, v.157, p.60-76, 2012.

MÉLO, A. S.; JUSTINO, F.; LEMOS, C. F.; SEDIYAMA, G. RIBEIRO, G. Suscetibilidade do ambiente a ocorrências de queimadas sob condições climáticas atuais e de futuro aquecimento global. Revista Brasileira de Meteorologia, v.26, n. 3, p.401-418, 2011.

MAVSAR, R.; CABÁN, A. G.; FARRERAS, V. The state of development of fire management decision support systems in America and Europe. Forest Policy and Economics, v.29, p.45-55, 2013.

NUNES, J. R. S. FMA + - um novo índice de perigo de incêndios florestais para o estado do Paraná - Brasil. Curitiba, 2005. 169 p. Tese (Doutorado em Engenharia Florestal) - Universidade Federal do Paraná, 2005.

NUNES, J. R. S.; FIER, I. S. N.; SOARES, R. V.; BATISTA, A. C. Desempenho da Fórmula de Monte Alegre (FMA) e da Fórmula de Monte Alegre alterada (FMA +) no Distrito Florestal de Monte Alegre. Floresta, 2010.

ROTHERMEL, R. C. A mathematical model for predicting fire spread in wildland fuels. Research Paper INT-115 Ogden: USDA Forest Service; 1972.

SAMPAIO, O. B. Análise da eficiência de quatro índices na previsão de incêndios florestais para a região de Agudos - SP. Curitiba, 1999. 157 p. Tese (Doutorado em Engenharia Florestal) - Universidade Federal do Paraná, 1999.

SCHUNK, C.; WASTL, C.; LEUCHNER, M.; MENZEL, A. Agricultural and Forest Meteorology Fine fuel moisture for site- and species-specific fire danger assessment in comparison to fire danger indices. Agricultural and Forest Meteorology. 2017; p. 234-235:31-47, 2017. 
TORRES, F. T. P.; RIBEIRO, G. A.; MARTINS, S. V.; LIMA, G. S. Mapeamento da suscetibilidade a ocorrências de incêndios em vegetação na área urbana de UbáMG. Revista Árvore, v.38, n.5, p.811-817, 2014.

TORRES, F. T. P.; LIMA, G. S.; MARTINS, S. V.; VALVERDE, S. R. Analysis of efficiency of fire danger indices in forest fire prediction. Revista Árvore, v.41(2), p.1-10, 2017.

van WAGNER, C. E. Development and structure of the Canadian forest fire weather index system. Forestry Technical Report 35 Ontario: Canadian Forest Service; 1987.

VIEGAS, D.X.; SOL, B.; BOVIO, B.; et al. Comparative study of various methods of fire danger evaluation in southern europe. International Conference on Forest Fire Research. (2.:1994: Coimbra). [Anais]... Coimbra.: [s.n.], 1994. P.571 590.

VIJAYAKUMAR, D. B. I. P.; RAULIER, F.; BERNIER, P. Y.; GAUTHIER, S.; BERGERON, Y.; POTHIER D. Lengthening the historical records of fire history over large areas of boreal forest in eastern Canada using empirical relationships. Forest Ecology and Management, v.347, p.30-39, 2015.

WHITE, B. L. A.; RIBEIRO, G. T. Análise da precipitação e sua influência na ocorrência de incêndios florestais no Parque Nacional Serra de Itabaiana, Sergipe, Brasil. Revista Ambiente \& Água, v.6, n.1, p. 48-156, 2011.

WHITE, L. A. S.; WHITE, B. L. A.; RIBEIRO, G. T. Evaluation of Forest Fire Danger Indexes for Eucalypt Plantations in Bahia, Brazil. International Journal of Forestry Research, v.2015, 6p., 2015.

ZUMBRUNNEN, T.; PEZZATTI, G. B.; MENÉNDEZ, P.; BUGMANN, H.; BÜRGI, M.; CONEDERA, M. Forest Ecology and Management, v. 261, p.2188-2199, 2011 